Article

\title{
(S)-10-Hydroxycamptothecin Inhibits Esophageal Squamous Cell Carcinoma Growth In Vitro and In Vivo Via Decreasing Topoisomerase I Enzyme Activity
}

\author{
Mengqiu Song $1,2,+$, Shuying Yin ${ }^{1,2, \dagger}$, Ran Zhao ${ }^{1,2}$, Kangdong Liu 1,2,3, Joydeb Kumar Kundu ${ }^{4}$, \\ Jung-Hyun Shim ${ }^{2,5}\left(\mathbb{D}\right.$, Mee-Hyun Lee ${ }^{1,2,3, *}$ and Zigang Dong ${ }^{1,2, *}$ \\ 1 Department of Pathophysiology, School of Basic Medical Sciences, Zhengzhou University, \\ Zhengzhou 450001, China; smq0705@126.com (M.S.); ysyyezi@163.com (S.Y.); zhaorpp1208@126.com (R.Z.); \\ kangdongliu@126.com (K.L.) \\ 2 China-US (Henan) Hormel Cancer Institute, No.127, Dongming Road, Jinshui District, Zhengzhou 450008, \\ China; s1004jh@gmail.com \\ 3 The Collaborative Innovation Center of Henan Province for Cancer Chemoprevention, \\ Zhengzhou 450001, China \\ 4 Li Ka Shing Applied Virology Institute, University of Alberta, Edmonton, AB T6G 2R3, Canada; \\ joydeb@ualberta.ca \\ 5 Department of Pharmacy, College of Pharmacy, Mokpo National University, Jeonnam 58554, Korea \\ * Correspondence: mhlee@hci-cn.org or mhyun_lee@hanmail.net (M.-H.L.); zgdong@hci-cn.org (Z.D.); \\ Tel.: +86-371-6558-7008 (M.-H.L.); +86-371-6558-7008 (Z.D.); Fax: +86-371-6558-7670 (M.-H.L.); \\ +86-371-6558-7670 (Z.D.) \\ + These authors contributed equally to this work.
}

Received: 28 October 2019; Accepted: 4 December 2019; Published: 6 December 2019

check for updates

\begin{abstract}
Topoisomerase (TOP) I plays a major role in the process of supercoiled DNA relaxation, thereby facilitating DNA replication and cell cycle progression. The expression and enzymatic activity of TOP I is positively correlated with tumor progression. Although the anticancer activity of (S)-10-Hydroxycamptothecin (HCPT), a TOP I specific inhibitor, has been reported in various cancers, the effect of HCPT on esophageal cancer is yet to be examined. In this study, we investigate the potential of HCPT to inhibit the growth of ESCC cells in vitro and verify its anti-tumor activity in vivo by using a patient-derived xenograft (PDX) tumor model in mice. Our study revealed the overexpression of TOP I in ESCC cells and treatment with HCPT inhibited TOP I enzymatic activity at $24 \mathrm{~h}$ and decreased expression at $48 \mathrm{~h}$ and $72 \mathrm{~h}$. HCPT also induced DNA damage by increasing the expression of H2A.X ${ }^{S 139}$. HCPT significantly decreased the proliferation and anchorage-independent growth of ESCC cells (KYSE410, KYSE510, KYSE30, and KYSE450). Mechanistically, HCPT inhibited the G2/M phase cell cycle transition, decreased the expression of cyclin B1, and elevated p21 expression. In addition, HCPT stimulated ESCC cells apoptosis, which was associated with elevated expression of cleaved PARP, cleaved caspase-3, cleaved caspase-7, Bax, Bim, and inhibition of Bcl-2 expression. HCPT dramatically suppressed PDX tumor growth and decreased the expression of Ki-67 and TOP I and increased the level of cleaved caspase- 3 and H2A.XS139 expression. Taken together, our data suggested that HCPT inhibited ESCC growth, arrested cell cycle progression, and induced apoptosis both in vitro and in vivo via decreasing the expression and activity of TOP I enzyme.
\end{abstract}

Keywords: topoisomerase I; (S)-10-Hydroxycamptothecin; esophageal squamous cell carcinoma; patient derived tumor xenograft (PDX) 


\section{Introduction}

Esophageal cancer (EC) is the eighth most common cancer globally and ranks sixth among the worst-prognosis cancers [1]. Clinically, EC is quite aggressive in nature and has a poor survival rate [1]. Esophageal squamous cell carcinoma (ESCC), the major subtype of EC, has a 79\% incidence in Asia [2]. Although the survival time of EC patients has improved in the past 30 years, it still remains poor compared to the other cancer types [3]. Because of apparently late diagnosis and the lack of a well-defined drug target, EC patients poorly respond to conventional chemoradiotherapy or chemoradiotherapy plus surgery. Thus, the identification of a valid drug target and the design of molecular target-based anticancer therapy would be a rational strategy to limit mortality from EC.

Topoisomerase I (TOP I) is an essential enzyme in both prokaryotes and eukaryotes [4]. TOP I is involved in the process of supercoiled DNA relaxation and the alleviation of the DNA helical constraints [5]. TOP I binds to the supercoiled DNA and subsequently, the cleaved one strand of the duplex DNA, then, a nick will be created at this site to allow the DNA to untwist and relax [6]. The participation of TOP I facilitates the process of DNA replication and the formation of RNA:DNA hybrids. The lack of TOP I or functional inactivation of the enzyme leads to DNA double-strand break (DSB) formation [7]. Elevated expression and enzymatic activity of TOP I have been reported to be closely related with cancer development, and it is a therapeutic target for the camptothecin (CPT) family [8]. The aberrantly high level of TOP I expression in paraffin-embedded tissues sections of different primary tumors including ovarian carcinoma and gastric cancer have been reported [9-13]. Thus, TOP I enzyme may be an ideal indicator of tumor progression and a potential drug for CPT family chemotherapeutic agents [14].

(S)-10-Hydroxycamptothecin (HCPT) (The chemical structure was indicated in Figure S1A), which is isolated from a Chinese tree Camptotheca cuminata, has been developed as a TOP I specific inhibitor [15]. HCPT has been reported to have broad anti-cancer activity in murine leukemia cells [16], human neuroblastoma [17], and colon cancer [18]. The nanosuspensions or polymorphic nanoparticle formulations of HCPT are the two new formulations that helped to increase the stability of the original HCPT and enhanced its anti-tumor efficacy $[19,20]$. Based on its broad anti-cancer spectrum, the evaluation of the effect of HCPT on ESCC cell proliferation and the capability of developing this compound for clinical management of EC would be a rational approach. Thus, the present study was designed to examine the effect of HCPT on the growth of ESCC cells' growth in culture as well as in vivo using a patient-derived xenograft (PDX) tumor model in mice. Here, we report that HCPT blunted the enzymatic activity of TOP I and the treatment with HCPT attenuated proliferation, arrested the cell cycle and induced apoptosis in ESCC cells, and diminished the growth of ESCC PDX models in vivo. Taken together, our study reveals the potential of HCPT as an anticancer therapeutic agent for ESCC.

\section{Results}

\subsection{TOP I Enzyme Acts as an Indicator of ESCC}

Since TOP I is a potential biomarker of tumor progression in many cancers, we performed immunohistochemical (IHC) analysis of TOP I expression in ESCC tissues. The expression of TOP I was significantly elevated in ESCC tumor tissues as well as in tumor-adjacent tissues as compared to normal tissues (Figure 1A,B). The transcripts of TOP1 also dramatically increased in esophageal cancer patients' tissues compared to normal tissues (Figure S1C) (Data obtained from http://gepia.cancer-pku.cn/). Data downloaded from the TCGA database showed that the TOP I gene is overexpressed in different stages of ESCC progression in patients as compared to its expression in normal tissues (Figure 1C). Moreover, patients with a high expression of TOP I had a relatively shorter overall survival time than those with low expression of the gene $(p=0.014)$ (Figure 1D) (Data obtained from http://gepia.cancerpku.cn/). Western blot was also performed to identify the expression of TOP I in cultured ESCC cells. The TOP I was highly expressed in most of the ESCC cell lines, especially in KYSE410, KYSE510, 
KYSE30, and KYSE450 cells, however its level was relatively low in normal esophageal epithelial cell SHEE (Figure 1E, Figure S5A).

A

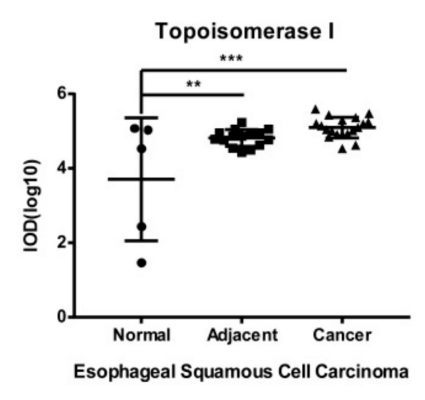

C
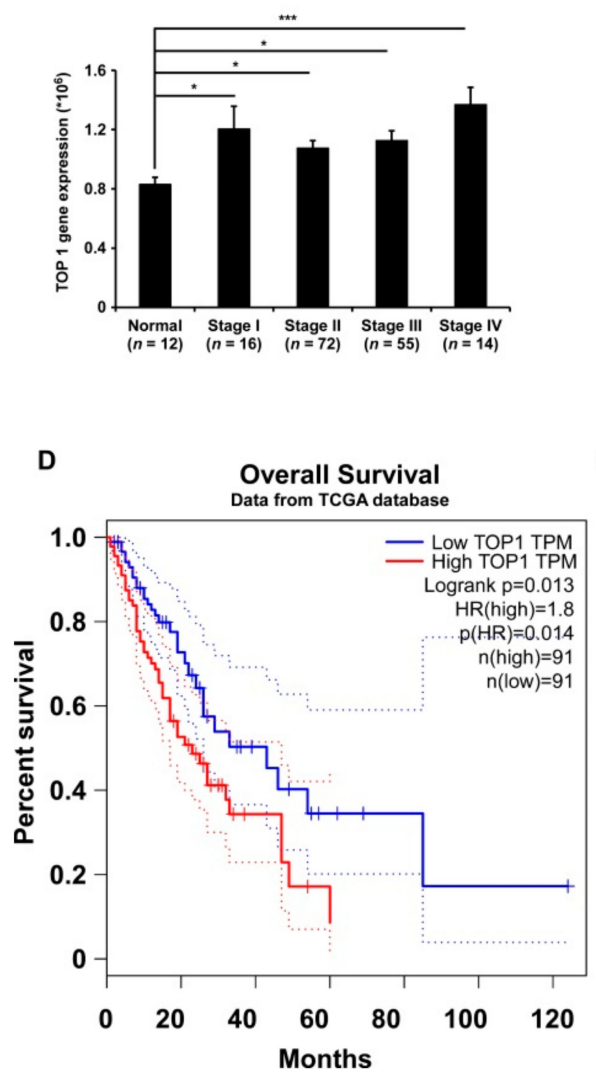

B

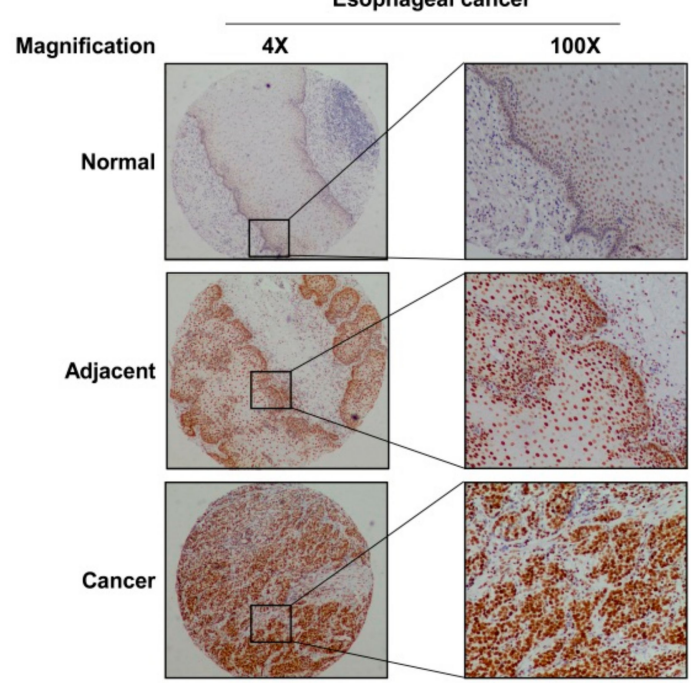

E

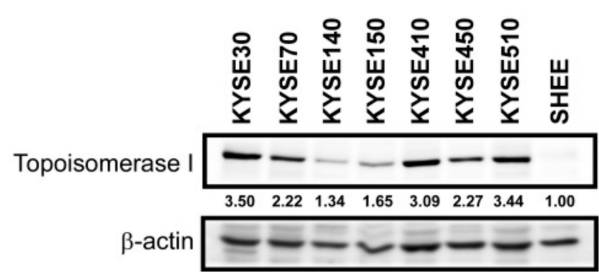

Figure 1. TOP I enzyme acts as an indicator of esophageal squamous cell carcinoma (ESCC). (A) Quantitation results of Topoisomerase (TOP) I immunohistochemical (IHC) staining on ESCC tissue array. Data was shown in the value of $\log 10$ (IOD). ${ }^{* *}, p<0.01 ;{ }^{* *}, p<0.001$ compared to normal tissues. (B) Images of IHC staining on esophageal normal (5 cases), adjacent (15 cases), and cancer (19 cases) tissues, separately (40× and 100× magnification). (C) TOP1 gene expression analysis in esophageal normal tissues and different stage cancer tissues (Data downloaded from TCGA database). $*, p<0.05 ;{ }^{* * *}, p<0.001$ compared to normal tissues. (D) Overall survival time of patients with high or low expression of TOP I gene (data obtained from http://gepia.cancer-pku.cn/). (E) The expression of TOP I in different kinds of ESCC cell lines was evaluated by Western blot assay. $\beta$-actin was used as an internal reference control.

\subsection{HCPT Inhibits the Proliferation of Esophageal Squamous Cell Carcinoma Cells}

In order to examine the effects of HCPT on ESCC cells, we selected four kinds of ESCC cell lines (KYSE410, KYSE510, KYSE30, and KYSE450), which contained higher levels of TOP I protein for cell proliferation assay (Figure 1E). The data indicated that HCPT treatment significantly decreased the proliferation of ESCC cells in a time- and concentration-dependent manner. The effective concentration 
( $\mathrm{EC}_{50}$ ) of HCPT ranged between $40 \mathrm{nM}$ and $320 \mathrm{nM}$ (Figure 2A). However, HCPT did not cause any cytotoxicity on normal esophageal epithelial cell SHEE (Figure S1B). Moreover, HCPT dramatically inhibited the foci formation at a concentration of $40 \mathrm{nM}$, which also showed significant inhibition of cell proliferation (Figure 2B,C). In the anchorage-independent cell growth assay, HCPT showed a strong inhibitory effect on colony formation consistent with MTT and foci assay in these ESCC cell lines (Figure 2D,E).
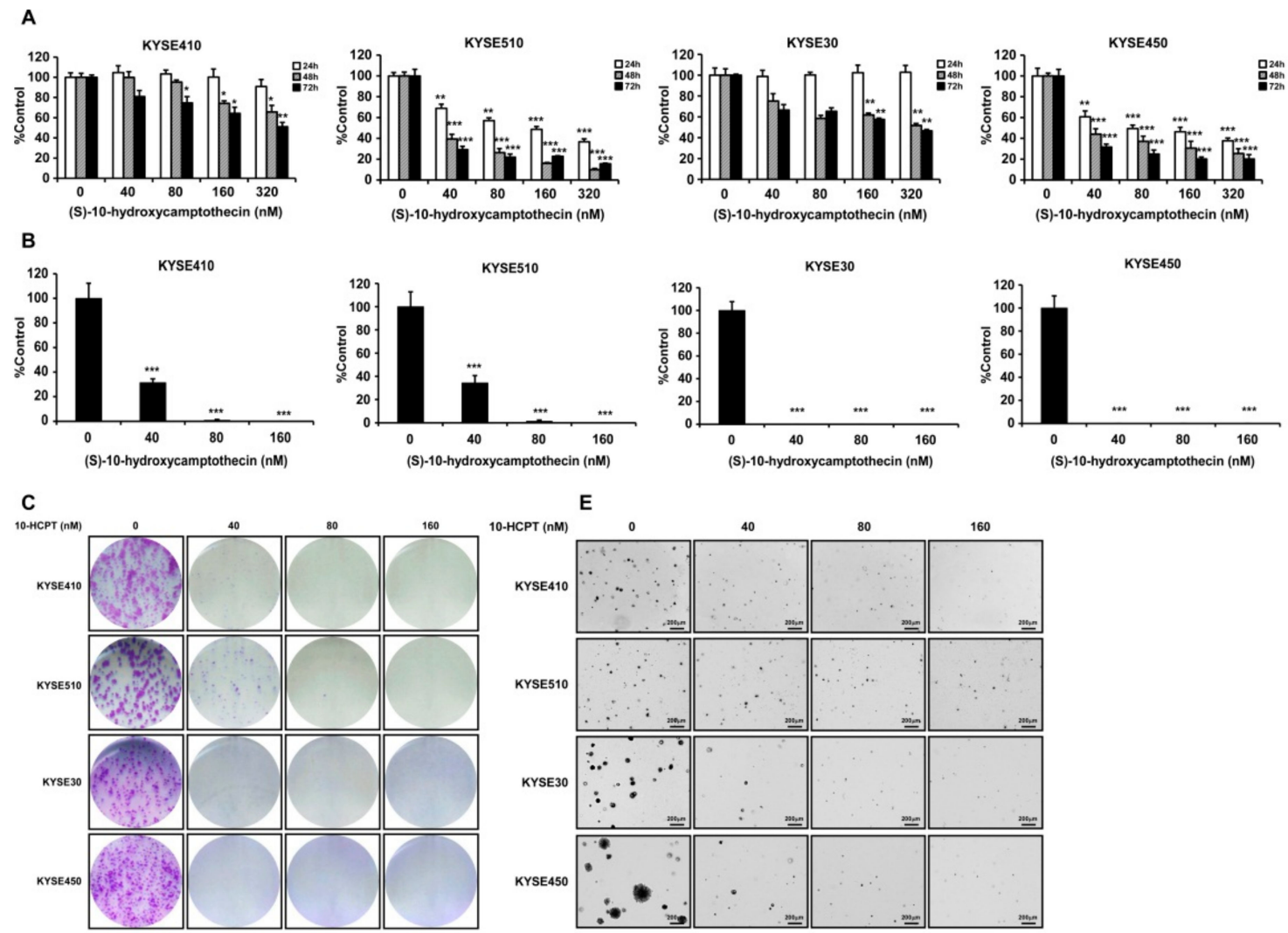

E
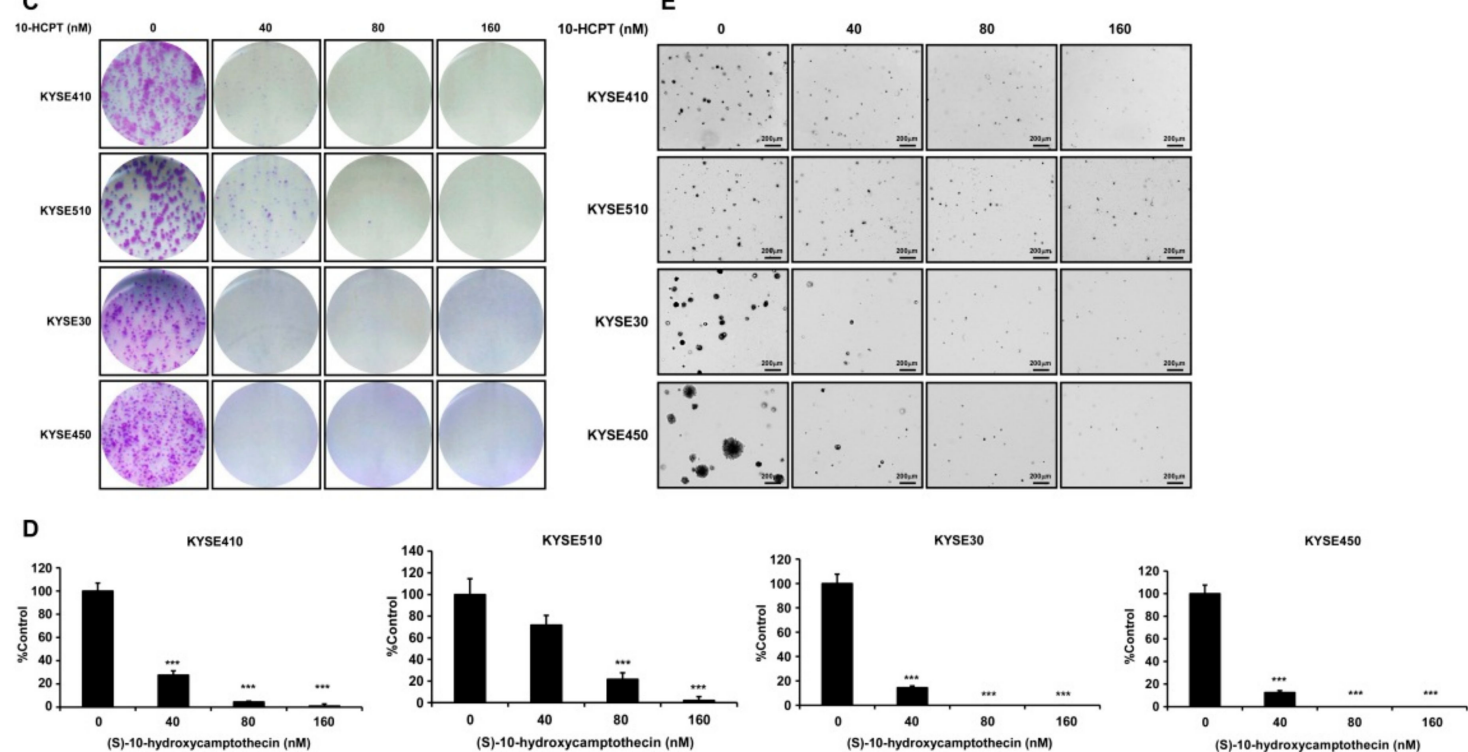

Figure 2. HCPT inhibits esophageal squamous cell carcinoma cells' proliferation. (A) Cells' proliferation of KYSE410, KYSE510, KYSE30, and KYSE450 post $\operatorname{HCPT~}(0,40,80,160$, and $320 \mathrm{nM})$ treatment were detected by MTT assay. Data were shown compared with the dimethyl Sulfoxide (DMSO) treated group. ${ }^{*}, p<0.05 ;{ }^{* *}, p<0.01 ; * * *, p<0.001$ compared to the controls. (B) Foci formation of ESCC cells were performed in 6-well plates with HCPT $(0,40,80$, and $160 \mathrm{nM})$ application for 7 days. The colonies number was analyzed and summarized, and the data were shown compared with the DMSO treated group. ${ }^{* *}, p<0.001$ compared to controls. (C) Images of crystal violet stained foci after HCPT $(0,40$, 80, and $160 \mathrm{nM}$ ) treatment for 7 days. (D) Anchorage-independent cell growth assay was performed to evaluate the effect of HCPT $(0,40,80$, and $160 \mathrm{nM})$ on cell growth. Colonies were captured and the number was counted after 3 weeks; the results are presented as treated group compared with the control group. ${ }^{* * *}, p<0.001$. (E) Representative pictures of colonies after HCPT treatment on KYSE410, KYSE510, KYSE30, and KYSE450 cells. Three independent repeats were performed for each experiment and were statistically analyzed. 


\subsection{HCPT Interrupts G2/M Cell Cycle Transition and Induces Apoptosis in ESCC Cells}

The incubation of KYSE410, KYSE510, KYSE30, and KYSE450 cells with HCPT led to cell cycle arrest at the G2/M phase (Figure 3A), inhibition of cyclin B1 expression, and elevation of p21 expression (Figure 3B, Figure S5B). Treatment with HCPT caused ESCC cells to undergo apoptosis as revealed by annexinV/PI staining. The total apoptosis cell number was collected based on early apoptosis (annexinV+/PI- gate) and late apoptosis (annexinV+/PI+ gate). HCPT significantly induced cell apoptosis compared to the DMSO treatment control, and this phenomenon happened both at early and late apoptosis (Figure 3C, Figure S2). Subsequent Western blot analysis showed changes in apoptosis markers in four candidate cell lines after HCPT treatment. The expression of cleaved PARP, cleaved caspase-3, cleaved caspase-7, $\mathrm{Bax}$, and Bim, which are markers of apoptosis, was significantly increased after HCPT treatment for $72 \mathrm{~h}$, while that of the anti-apoptotic protein Bcl-2 was markedly decreased (Figure 3D, Figure S6).
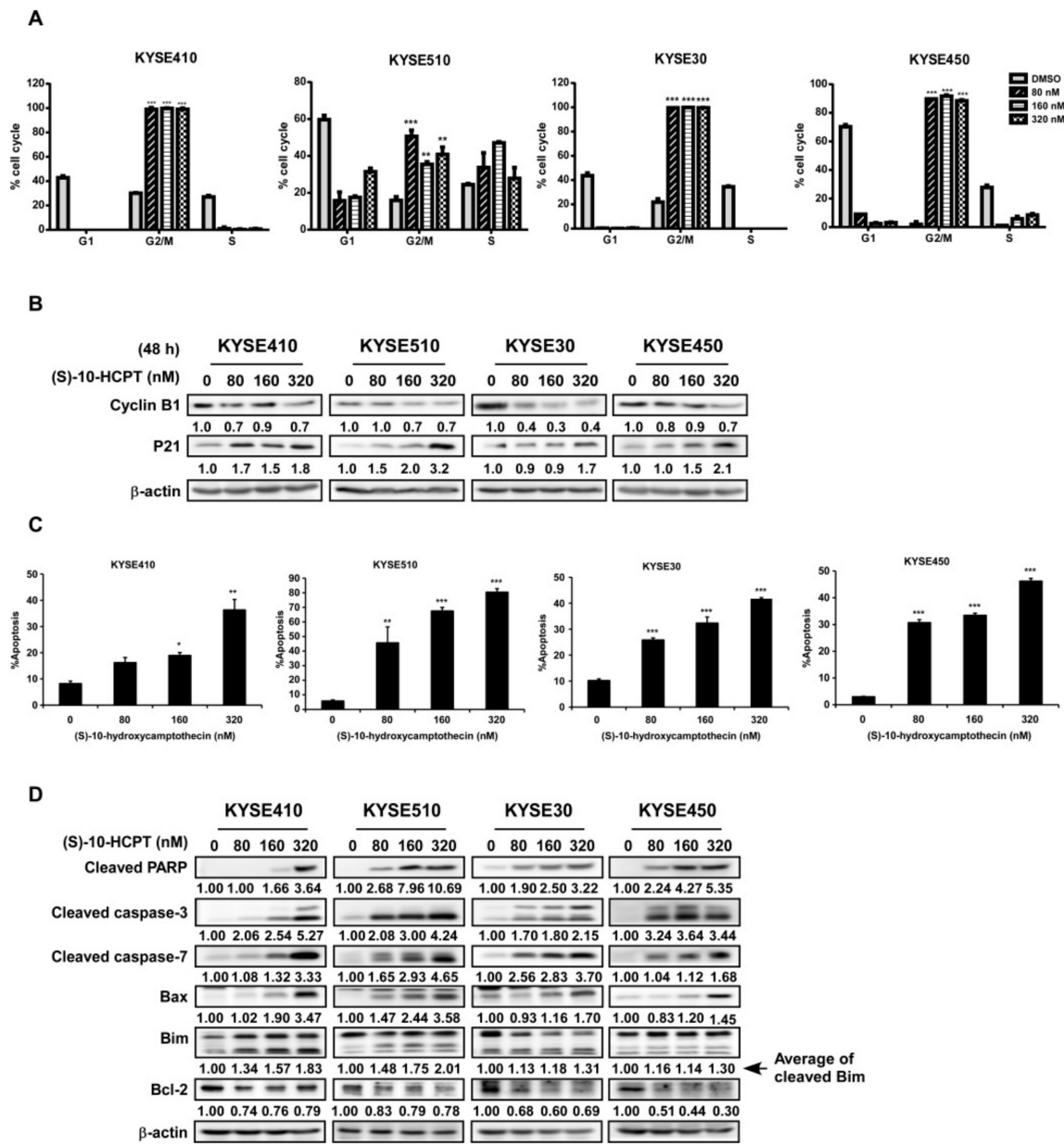

Figure 3. HCPT interrupts ESCC cells' G2/M cell cycle transition and induces apoptosis. (A) Cell cycle analysis by flow cytometry on ESCC cells after $\operatorname{HCPT}(0,80,160$, and $320 \mathrm{nM})$ treatment for $12 \mathrm{~h}$. Statistics of cell cycle distribution were shown as \% cell cycle. ${ }^{* *}, p<0.01$; ${ }^{* * *}, p<0.001$ compared to DMSO controls. (B) Western blotting analysis to detect the expression of G2/M phase markers cyclin B1 and p21 after HCPT $(0,80,160$, and $320 \mathrm{nM})$ treatment in ESCC cells. $\beta$-actin was used as an internal reference control. (C) Cell apoptosis were checked by flow cytometry using annexin V and the PI double staining method. The results were summarized and shown as $\%$ apoptosis. ${ }^{*}, p<0.05$; **, $p<0.01$; **, $p<0.001$ compared to the controls. (D) Cells were treated with HCPT $(0,80,160$, and $320 \mathrm{nM}$ ) for $72 \mathrm{~h}$. Lysates were harvested and analyzed by Western blotting for the expression of apoptosis markers as indicated. $\beta$-actin was used as an internal reference control. Three independent repeats were performed for flow cytometry detection and were statistically analyzed. 


\subsection{HCPT Decreases TOP I Enzyme Activity and Inhibits its Expression in ESCC}

We next assessed the effect of HCPT on the TOP I enzyme activity in ESCC. DNA electrophoresis showed that the amount of supercoiled DNA was obviously increased as compared to the control group in all of the four ESCC cell lines after treatment of HCPT for $24 \mathrm{~h}$ (Figure 4A). The data indicated that HCPT treatment significantly inhibited the activity of TOP I enzyme and interrupted the re-ligation of DNA strand. Western blot analysis of lysates from ESCC cells treated with HCPT for $48 \mathrm{~h}$ and $72 \mathrm{~h}$ showed decreased TOP I expression in all of the four candidate cell lines (Figure 4B, Figure S7A,B). We also found that the expression of rH2A.X was elevated $48 \mathrm{~h}$ post HCPT treatment as compared with the DMSO-treated group, indicating that DNA damage occurred because of the lack of sufficient TOP I activity (Figure 4C, Figure S7C).

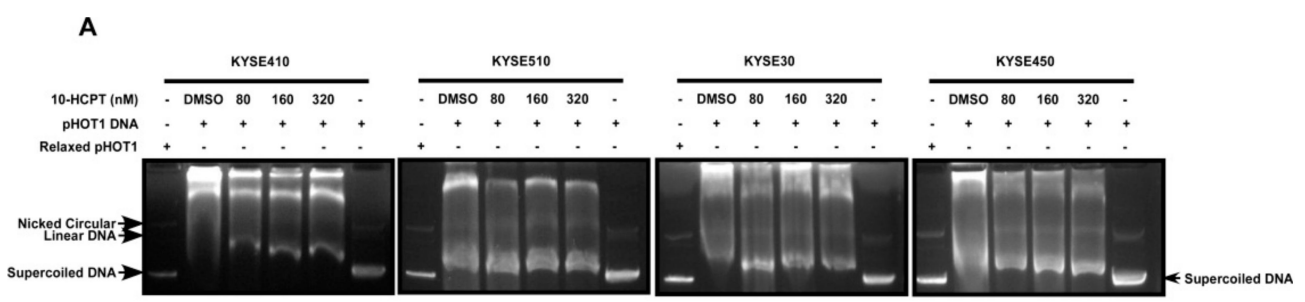

B

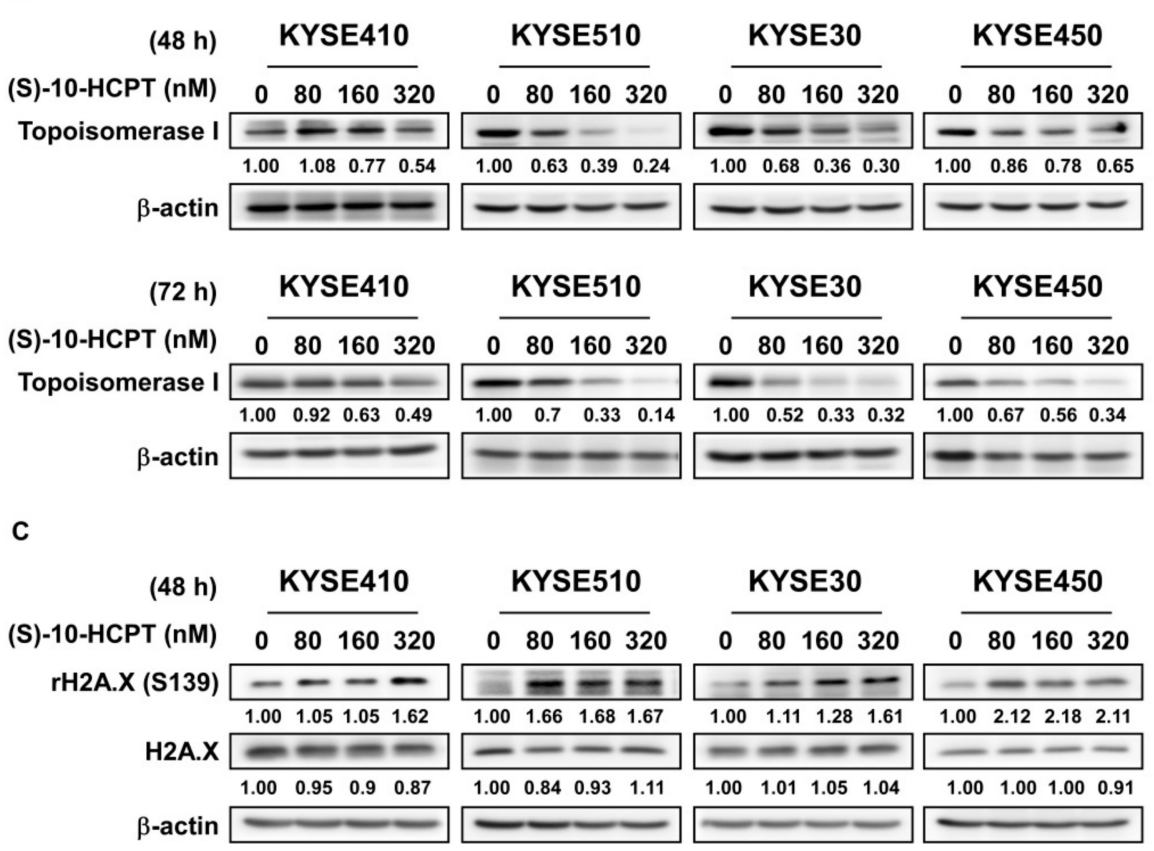

Figure 4. HCPT decreases TOP I enzyme activity and inhibits its expression in ESCC. (A) Cell lysates were analyzed after HCPT treatment with the indicated concentration for $24 \mathrm{~h}$. TOP I enzyme was extracted following the producer's protocol (www.topogen.com). The activity of TOP I was measured and visualized by DNA electrophoresis as the indicated amount of supercoiled DNA. (B) ESCC cells were harvested and lysated with HCPT treatment for 48 and $72 \mathrm{~h}$ and the expression of TOP I was visualized by Western blot method. $\beta$-actin was used as an internal reference control. (C) Phosphorylation of rH2A.X at serine 139 site indicated DNA foci formation and damage, which is the late-stage phenomenon after TOP I activity was broken down. Western blot was carried out for developing the expression of H2A.XS139 after HCPT treatment at $48 \mathrm{~h}$ in the indicated concentration. $\beta$-actin was used as an internal reference control. Three independent repeats were performed for each experiment. 


\subsection{HCPT Attenuated ESCC PDX Tumor Growth in Mice}

PDX tumors in mice are an experimental model for pre-clinical study to assess the potential of anti-tumor activity of putative drug molecules [21]. To examine the possible inhibitory effects of HCPT on PDX tumor growth, patient-derived LEG104 and LEG110 ESCC tumors with high expression levels of TOP I were adopted in this investigation. The treatment of PDX tumors grown in mice with HCPT ( $4 \mathrm{mg} / \mathrm{kg}$ and $8 \mathrm{mg} / \mathrm{kg}$ body weight) decreased the tumor volume and tumor weight significantly (Figure 5A-D). The weight and size of tumors were also dramatically decreased after HCPT administration for 18 or 29 days (Figure 5B,C,E,F). While eliciting antitumor activity in PDX tumor-bearing mice, HCPT application did not exhibit any remarkable signs of toxicity in mice (Figure S3). The IHC and HE staining of the PDX tumor sections showed more regression of tumor cells and changes in morphology, such as cell enlargement, caryolysis, variable nucleus size, nuclear pyknosis, and nucleolar margin blurring in the treatment group in comparison with the vehicle group (Figure 5G). Darker staining in the cytoplasm by eosin in the HCPT-treated groups indicated apoptosis phenomenon which was not evident in the vehicle group (Figure 5G). Moreover, there was a significant decrease in the expression of Ki-67 and elevation in cleaved caspase-3, which indicated that the cell proliferation was inhibited and apoptosis was induced after HCPT application. The IHC analysis of HCPT-treated tumors also showed decreased TOP I expression and increased phosphorylated rH2A.X level (Figure 5G,H, Figure S4).

\section{Discussion}

The aberrant elevated expression of DNA TOP I at both the protein and mRNA levels has been frequently observed in several human malignancies compared to normal tissues. Therefore, making TOP I a potential target for anticancer medicines is important [14]. TOP I protein level has been reported to be highly expressed in various ESCC cell lines and the protein expression index demonstrated a significant correlation with the $\mathrm{IC}_{50}$ value of camptothecin-11 treatment, which did not show any correlation with the mRNA level of TOP I [22]. We examined the effects of 10-HCPT, a derivative of camptothecin, on the growth of ESCC cells in culture cells as well as in vivo and elucidated its underlying mechanism. In our findings, IHC results of ESCC tissue array indicated that TOP I protein was overexpressed in tumors and tumor-adjacent tissues compared with normal esophageal epithelial tissues (Figure 1A,B). This finding was in good agreement with the elevated expression of TOP I in ESCC cells in culture (Figure 1E, Figure S5A). Meanwhile, the GEPIA database indicated that the transcripts of TOP1 were significantly increased in ESCA patient samples (Figure S1C). The positive correlation between TOP I expression and the ESCC cancer stage, especially stage IV (Figure 1C), make this gene a potential target for developing therapy for ESCC. Patient survival time also exhibited significant difference between the TOP I-high and TOP I-low expression groups, demonstrating that high TOP I expression leads to poor outcomes. 
A

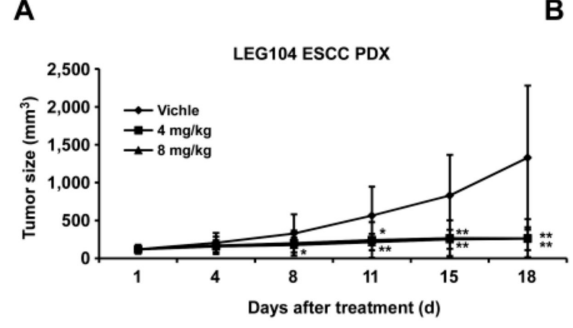

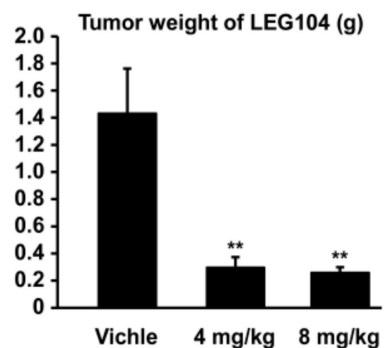

C

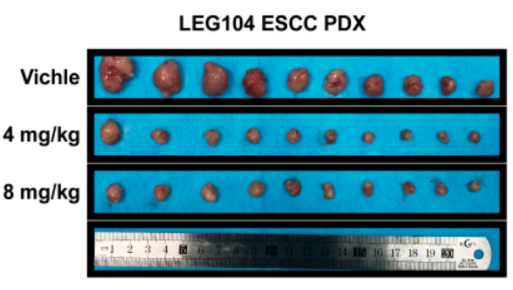

E

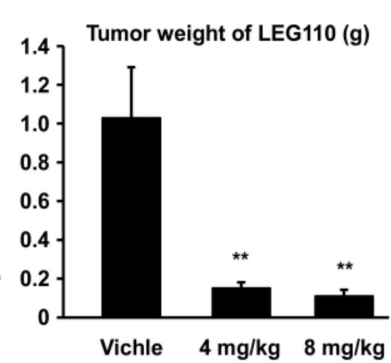

F

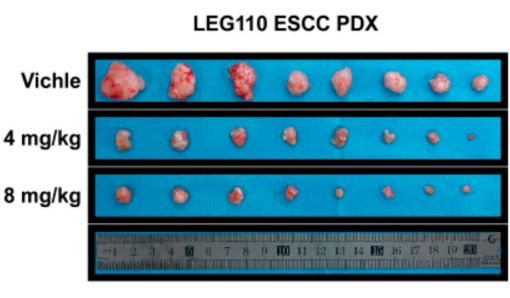

G

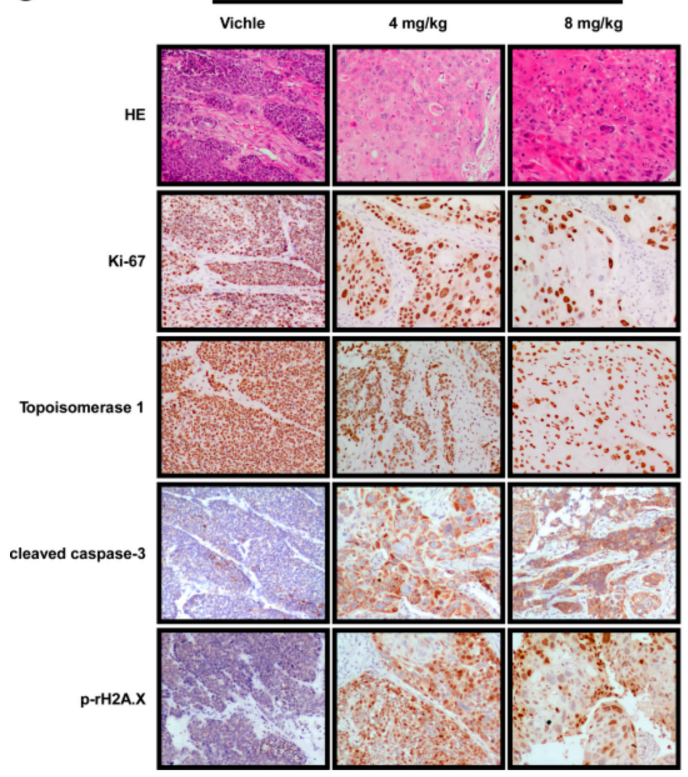

H

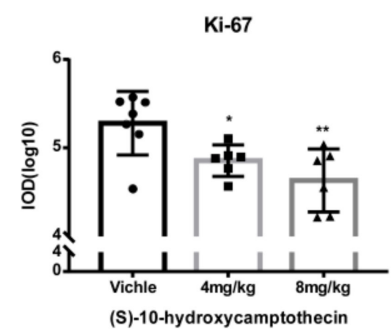

Cleaved caspase- 3

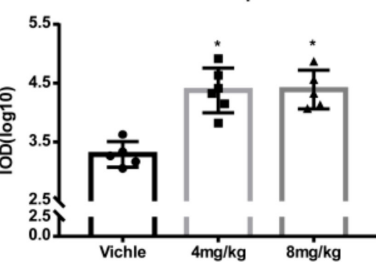

(S)-10-hydroxycamptothecin

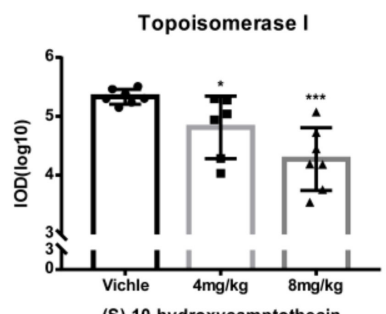

(S)-10-hydroxycamptothecin

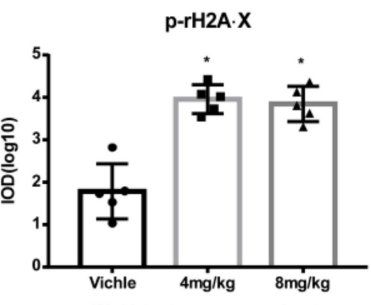

(S)-10-hydroxycamptothecin

Figure 5. HCPT attenuated ESCC patient-derived xenograft growth in vivo. The effect of (S)-10-Hydroxycamptothecin on the growth of ESCC PDX was plotted over 18 days (HEG104) (A) and 29 days (LEG110) (D). Vehicle or HCPT (4 or $8 \mathrm{mg} / \mathrm{kg}$ ) was administered by paraneoplastic injection twice weekly (10 (for LEG104) or 8 (for LEG110) mice per group). The tumor volume was also measured twice weekly. ${ }^{*}, p<0.05 ;{ }^{* *}, p<0.01$ indicates the significant decrease of tumor volume compared to the vehicle group. Data are shown as mean value $\pm \mathrm{SE}$. The tumor weight was recorded when the mice were sacrificed (B,E). The asterisks ${ }^{* *}, p<0.01$ indicates the significant decrease of body weight. The photographs show PDX tumors isolated from mice treated with HCPT $(\mathbf{C}, \mathbf{F})$. (G) IHC was performed for measuring the expression of Ki-67, TOP I, cleaved caspase-3, and H2A.XS139 in PDX tissue specimens; 5 to 7 independent tissue specimens were adopted for each group and analyzed (100× magnification). (H) Quantification results of responding IHC results. IOD values were measured by Image-pro Plus 6.0 software and shown as values \pm SD. ${ }^{*}, p<0.05 ;{ }^{* *}, p<0.01 ;{ }^{* * *}, p<0.001$ indicate a significant decrease in the indicated proteins in the treated tissues compared to the controls. 
TOP I was reported to be a molecular target of chemotherapeutic medicine of the camptothecin (CPT) family [8]. Compared to CPT, HCPT showed more profound anti-cancer effects with relatively less toxicity. According to several other studies, HCPT exhibited a broad spectrum of anti-cancer effect in a variety of solid tumors in vitro and in vivo [23-25]. There are also other camptothecin analogues that were used to inhibit esophageal squamous cell carcinoma growth, like gimatecan and irinotecan. Compared with the first-line clinical agent, irinotecan, gimatecan showed stronger anticancer activity. Both gimatecan and irinotecan functioned through topoisomerase inhibition and subsequent induction of DNA damage, S-phase cell cycle arrest, and induction of apoptosis [26]. In a phase III clinical trial, the progression-free survival (PFS) $(p=0.006)$ and objective response rate (ORR) $(p=0.002)$ of recurrent or metastatic ESCC were prolonged in the irinotecan plus intravenous infusion of irinotecan (S-1) $\left[160 \mathrm{mg} / \mathrm{m}^{2}\right.$ ] group compared to the S-1 monotherapy group, leading to the possibility of TOP I inhibitor on ESCC clinical treatment [27]. HCPT was reported to decrease cell proliferation and induce apoptosis or autophagy in colon cancer cells [18] and non-small cell lung cancer (NSCLC) cells [28]. In agreement with these reports, we found that HCPT suppressed ESCC cells proliferation dramatically in a time- and concentration-dependent manner in both the MTT assay and foci assay (Figure 2A-C). The anti-tumor effect of HCPT was further supported by decreased size and number of colonies in anchorage-independent cell growth assay (Figure 2D,E). It was reported in earlier studies that HCPT induced $S$ phase cytotoxicity via inducing replication fork collision and finally leading to G2/M phase cell cycle arrest $[15,29]$. We confirmed the finding through flow cytometry analysis of the cell cycle after HCPT treatment for $24 \mathrm{~h}$. HCPT application destroyed S phase cell cycle progression and inhibited the G2/M phase of cell cycle transition in ESCC cell lines, attenuated cyclin B1 protein expression, as well as increased the expression of P21 protein (Figure 3A,B, Figure S5B). Our flow cytometry analysis results of the induction of apoptosis in ESCC cells upon treatment with HCPT (Figure 3C, Figure S2) was also well correlated with previous reports of apoptosis induction by HCPT in SMS-KCNR human neuroblastoma cells [17], human osteosarcoma cells [30], colon cancer cells [18], and hepatoma Hep G2 cells [31]. The elevated expression of cleaved PARP, cleaved caspase-7, cleaved caspase-3, and Bax and the decreased expression of Bcl-2 were the molecular probes to the apoptosis induction by HCPT. Moreover, the decreased expression of long form of Bim or the increased expression of short form of Bim also indicated apoptosis post HCPT treatment (Figure 3D, Figure S6).

Accumulating data indicated that HCPT formed a complex with DNA and TOP I, resulting in double strand DNA breakage and cell death directly [15,32]. Since human TOP I is essential for topological stress releasing and topology modulation, the damage of TOP I function will lead to supercoiled DNA because the DNA strands cannot release superhelix during transcription and replication [4]. Thus, the amount of supercoiled DNA will reflect the enzyme activity of TOP I in double strand DNA superhelix locally unwinding. Treatment of cells for $24 \mathrm{~h}$ with HCPT as a specific inhibitor of TOP I showed obvious supercoiled DNA formation compared to the DMSO control group in ESCC cells (Figure 4A). This finding demonstrated that the function of human TOP I activity was diminished after HCPT treatment. Besides decreasing the TOP I activity, HCPT treatment also reduced the protein expression of TOP I in ESCC cells as revealed by immunoblot analysis (Figure 4B, Figure S7A). This decrease in TOP I protein expression was more obvious at $72 \mathrm{~h}$ in ESCC cells treated with HCPT (Figure 4B, Figure S7B).

Histone H2A.X is a variant histone that is responsible for DNA-damage repair [33]. Its phosphorylation at Ser139 is required for the recognition of double-stranded breaks (DSBs) [34]. Histone H2A. $X^{S 139}$ was indicated to be characteristic of higher genome instability and its increase in protein expression indicates DNA double-strand breaks and DNA repair emergency [35]. Due to the important function of TOP I in DNA and chromatin conformations, the application of the camptothecin family inhibitor finally induced cellular DNA damage and cell death [36]. Based on this knowledge, we assessed any possible changes in H2A. $X^{\mathrm{S} 139}$ expression following the administration of HCPT. The exposure of ESCC cells to HCPT for $48 \mathrm{~h}$ increased the H2A.X ${ }^{S 139}$ level, which indicated the enhancement of DNA damage after the inhibitor application (Figure 4C, Figure S7C). 
The patient-derived xenograft (PDX) tumors reflect the gene expression profiles and epigenomes of the original tumors [37], making the xenograft an ideal model for anti-cancer medicine screening. In our study, ESCC PDX LEG104 and LEG110 were used to evaluate the chemotherapeutic ability of HCPT. The administration of HCPT inhibited ESCC PDX tumor growth in both of ESCC cases, and the tumor weight was dramatically decreased (Figure 5A-F). However, HCPT treatment did not show remarkable changes in mouse body weight and pathological changes in the liver and spleen (Figure S3). Immunohistochemical analysis of PDX xenograft tumor tissues from HCPT- or vehicle-treated groups also showed reduced expression of Ki-67 and TOP I and increased expression of cleaved caspase-3 and H2A.X ${ }^{S 139}$ in HCPT-treated tumors (Figure 5G,H, Figure S4), further supporting the anti-proliferative and apoptosis inducing effect of HCPT on ESCC. Of note, the animal experiments in our study are still limited for statistical assessment, however it indicated the way for ESCC clinical treatment. The activity of TOP I enzyme was inhibited as an early stage phenomenon after HCPT administration and the DNA damage, cell cycle arrest, and apoptosis induction were subsequently triggered as a final consequence in regressing the size of PDX tumors.

In conclusion, TOP I is highly expressed in human ESCC tumors and tumor-adjacent tissues and treatment with HCPT attenuates cell proliferation, inhibits G2/M phase cell cycle transition, and induces apoptosis in ESCC cells in culture via inhibition of the expression and activity of TOP I enzyme (Figure 6). Moreover, HCPT suppresses ESCC PDX tumor growth in vivo, which is associated with reduced expression of TOP I and other cell proliferation markers and elevated expression of apoptosis markers. Thus, TOP I appears as a valid anticancer drug target and HCPT, as an inhibitor of TOP I, may be considered for further clinical development for ESCC therapy.

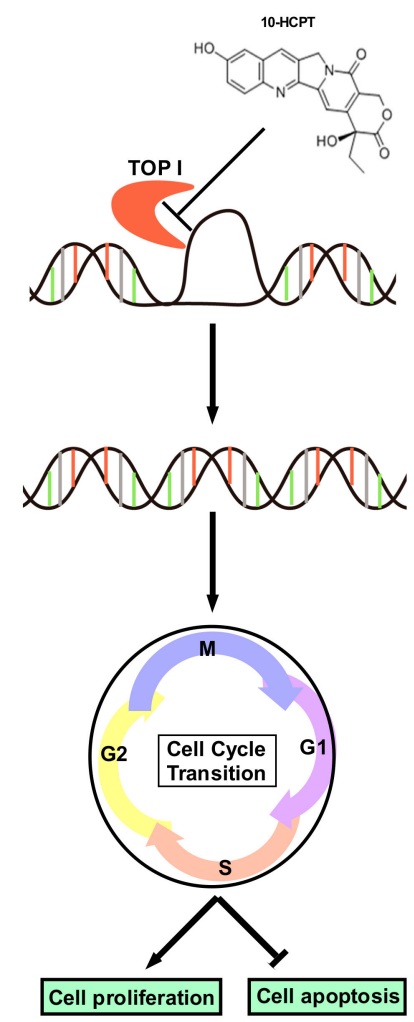

Figure 6. Schematic diagram of the mechanism of HCPT on ESCC. HCPT blocked the combination between TOP I enzyme and supercoiled DNA, decreasing the enzyme activity of TOP I. Foci formation subsequently occurred to promote DNA damage. As a consequence, cell cycle transition was inhibited and the G2/M phase was arrested. All of these processes finally led to cell proliferation halt and cell apoptosis, as indicated by the decreasing Ki-67 expression as well as the changing apoptosis markers. 


\section{Materials and Methods}

\subsection{Reagents}

HCPT (purity > 98\%) was purchased from Meilun Biotechnology Co., Ltd. (CAS: 19685-09-7, Dalian, China) and was analyzed and authenticated by high-performance liquid chromatography. Thiazolyl blue tetrazolium bromide (MTT) powder and Crystal violet powder were purchased from Solarbio Technology Co., Ltd. (Beijing, China). TOP I antibody was purchased from Abcam and antibodies to detect apoptosis such as: cleaved PARP (Cat: 9664), cleaved caspase-3 (Cat: 5625), cleaved caspase-7 (Cat: 8438), Bim (Cat: 2933), Bax (Cat: 5023), and Bcl-2 (Cat: 15071) as well as p21 (Cat: 2947) were purchased from Cell Signaling Technology (Beverly, MA, USA).

\subsection{Cell Culture}

KYSE410, KYSE510, KYSE30, and KYSE450 human esophageal cancer cell lines were purchased from the Type Culture Collection of the Chinese Academy of Sciences (Shanghai, China). Normal human immortalized esophageal epithelial cell SHEE was donated by Dr. Enmin Li in the Laboratory of Tumor Pathology (Shantou University Medical College, Shantou, Guangdong, China) [38]. The cells were cultured in RPMI-1640 contained with streptomycin $(100 \mu \mathrm{g} / \mathrm{mL})$, penicillin (100 units/mL), and 10\% FBS (BI, Kibbutz, Israel) in an incubator with $5 \% \mathrm{CO}_{2}$ and $37{ }^{\circ} \mathrm{C}$ atmosphere. SHEE (N1217) was donated by Dr. Enmin Li (Laboratory of Tumor Pathology, Shantou University Medical College, Shantou, Guangdong, China). All of the cells had passed the STR profiling and had been identified as correct cell lines. Each vial of frozen cells was thawed and maintained in culture within 10 passages.

\subsection{MTT Assay}

Cells were seeded in 96-well plates for MTT assay according to cell growth characteristics $\left(3 \times 10^{3}\right.$ cells per well for KYSE410, $1.5 \times 10^{3}$ cells per well for KYSE510, $2 \times 10^{3}$ cells per well for KYSE30, $1.5 \times 10^{3}$ cells per well for KYSE450). Cells were treated with different concentrations of HCPT or vehicle. After incubation for 24,48 , and $72 \mathrm{~h}$, cell proliferation was measured by MTT $(0.5 \mathrm{mg} / \mathrm{mL})$ reagent, separately.

\subsection{Foci Formation Assay}

KYSE410, KYSE510, KYSE30, and KYSE450 cells were used to perform Foci formation assay following the protocols described below. Cells $\left(1 \times 10^{3}\right.$ cells/well) were seeded into 6-well plates and incubated for $24 \mathrm{~h}$. The cells were then treated with different concentrations of HCPT or vehicle for another 7 days. The cell culture media was changed every three days. Foci were washed by PBS and then fixed and stained with $0.5 \%$ crystal violet solution.

\subsection{Anchorage-Independent Cell Growth}

Agar mix (0.5\%) was prepared to form a base layer, which contained vehicle and HCPT $(40,80$, and $160 \mathrm{nM})$ in the 6-well plate. Then, cells $\left(8 \times 10^{3}\right.$ cells/well) suspended in complete medium were added to $0.3 \%$ agar with vehicle, 40,80 , and $160 \mathrm{nM} \mathrm{HCPT} \mathrm{in} \mathrm{a} \mathrm{top} \mathrm{layer} \mathrm{over} \mathrm{the} \mathrm{base} \mathrm{layer.} \mathrm{Each} \mathrm{of}$ the agar plates were cultured and maintained in the incubator for 3 weeks and pictures were taken at the third week by microscope. Then, colonies were counted and summarized using the Image-Pro Plus software (v.6.0) program (Media Cybernetics, Silver Spring, MD, USA).

\subsection{Cell Cycle Analysis}

Cells $\left(3.5 \times 10^{5}\right.$ cells per dish for KYSE410, $2 \times 10^{5}$ cells per dish for KYSE510, KYSE30, KYSE450) were seeded into 6-well plates and incubated for $24 \mathrm{~h}$. Then, media was replaced with FBSand-antibiotic-free fresh RPMI-1640 media for $12 \mathrm{~h}$. Different concentrations of HCPT or vehicle were added to the cells and incubated for $48 \mathrm{~h}$, then the cells were harvested together with supernatant by 
trypsin digestion. The cells were fixed with $70 \%$ ethanol at $-20{ }^{\circ} \mathrm{C}$ overnight and washed by pre-cold PBS and then re-suspended with $250 \mu \mathrm{L} 0.6 \%$ Triton X-100 solution. Cells were then incubated with RNase A $(200 \mu \mathrm{g} / \mathrm{mL})$ for $1 \mathrm{~h}$ at room temperature followed by cell cycle analysis using a BD FACSCalibur Flow Cytometer (BD Biosciences, San Jose, CA, USA) after propidium iodide (PI, $20 \mu \mathrm{g} / \mathrm{mL}) \mathrm{staining}$ for $15 \mathrm{~min}$ at $4^{\circ} \mathrm{C}$.

\subsection{Annexin V Apoptosis Assay}

Cells $\left(2 \times 10^{5}\right.$ cells per dish for KYSE410, $1 \times 10^{5}$ cells per dish for KYSE510, KYSE30, and KYSE450) were seeded into 6-well plates. After incubation for $24 \mathrm{~h}$, cells were treated with HCPT $(0,80,160$, or $320 \mathrm{nM}$ ) or vehicle for $72 \mathrm{~h}$ and harvested together with cell culture media for apoptosis detection. Harvested cells were then incubated with Annexin V binding buffer for $10 \mathrm{~min}$ at room temperature followed by PI staining in dark atmosphere. Apoptosis was detected by a BD FACSCalibur Flow Cytometer (BD Biosciences, San Jose, CA, USA).

\subsection{Western Blotting}

Protein from KYSE410, KYSE510, KYSE30, and KYSE450 cells treated with or without HCPT were extracted using NP-40 cell lysis buffer (50 mM Tris pH 8.0, $150 \mathrm{mM} \mathrm{NaCl}, 0.5-1 \%$ NP-40, Protease inhibitor cocktail, dephosphorylate inhibitor tablets, and $1 \mathrm{mM}$ phenylmethylsulfonyl fluoride (PMSF)). Bichinconinic acid (BCA) assay kit (Solarbio, Beijing, China) was used for protein quantification and the same amount of protein were prepared for each sample according to the protein concentration. Sodium dodecyl sulphate-polyacrylamide gel electrophoresis (SDS-PAGE) was performed to separate different proteins and then the proteins were transferred onto polyvinylidene fluoride membranes, followed by blocking with $5 \%$ non-fat milk for $1 \mathrm{~h}$ at room temperature. Subsequently, appropriate primary antibodies were incubated at $4^{\circ} \mathrm{C}$ overnight, followed by incubation with appropriate HRP-linked secondary antibodies for $2 \mathrm{~h}$ at room temperature. Blots were visualized by the enhanced chemiluminescence (ECL) detection reagent.

\subsection{Immunohistochemical (IHC) Analysis}

Paraffin-embedded sections of human esophageal squamous cell carcinoma tissue microarray and PDX tumors from mice were subjected to IHC analysis. About $5 \mu \mathrm{m}$ of thickness of the sections were prepared for each tumor sample. The specimens were blocked with 5\% goat serums following antigen unmasking by sodium citrate solution and were first incubated with appropriate primary antibodies at $4^{\circ} \mathrm{C}$ overnight, followed by secondary antibodies for $2 \mathrm{~h}$ at room temperature. The sections were then incubated with 3,3'-diaminobenzidine (DAB) solution to visualize the staining of the target proteins by counterstaining with hematoxylin, dehydrating through a graded series of alcohol into xylene, and being mounted under glass coverslips.

\subsection{TOP I Assay}

This experiment was performed following the protocol of TOP I assay kit (TopoGEN, Florida, USA). Cells treated with HCPT or vehicle for $48 \mathrm{~h}$ were harvested together with media and dispersed cell pellets by TEMP buffer (10 mM Tris-HCL, pH = 7.5, $1 \mathrm{mM}$ EDTA, $4 \mathrm{mM} \mathrm{MgCl}$, $0.5 \mathrm{mM}$ PMSF). Nuclear pellets were re-suspended with TEP buffer (same as TEMP but lacking $\mathrm{MgCl}_{2}$ ) following homogenizing and centrifuging. Then, equal volume of $1 \mathrm{M} \mathrm{NaCl}$ was added to each tube to get the supernatant by ultracentrifuge. Then, the TOP I activity assay was performed using all of the reactants containing DNA, test extract, Tris-Glycine-SDS (TGS) buffer, and $\mathrm{H}_{2} \mathrm{O}$ to reach a final volume of $20 \mu \mathrm{L}$. The mixture was incubated at $37^{\circ} \mathrm{C}$ for $30 \mathrm{~min}$ and the reaction was terminated by adding $5 \times$ stop buffer. The samples were directly loaded onto a $1 \%$ agarose gel for electrophoresis until the dye front of bromophenol blue was about 70\% down the gel. The gel was then stained with Ethidium bromide (EB) solution for $15 \mathrm{~min}$ and then performed photo-documentation immediately. 


\subsection{PDX Tumor Model in Mice}

Six week old female severe combined immunodeficient (SCID) mice (Vital River Labs., Beijing, China) were adopted in this study. Tumor tissues from patients were implanted subcutaneously on the mice and the mice were divided into 3 groups ( $n=10$ /group) when the tumor mass reached an average volume of $100 \mathrm{~mm}^{3}$. The mice in different groups were treated with vehicle or HCPT $(4 \mathrm{mg} / \mathrm{kg}$ or $8 \mathrm{mg} / \mathrm{kg}$ ), respectively, twice per week by paraneoplastic injection. The injectable suspension of HCPT was made by mixing HCPT with 10\% DMSO, 40\% PEG-400, and 50\% PBS. The tumor volume of each mice was measured twice per week and then calculated by the following formula: tumor volume $\left(\mathrm{mm}^{3}\right)=$ length $\times$ width $\times$ height $\times 0.52$. Mice were euthanized and tumors were extracted until the average tumor volume reached $1000 \mathrm{~mm}^{3}$. This study was approved by the Ethics Committee of Zhengzhou University (Zhengzhou, Henan, China) (ZZUHCI-2019012) and the patients whose tumor samples were used in this study were completely informed and provided consent.

\subsection{Statistical Analysis}

Data in this paper were shown as mean \pm standard deviation (SD). Student's $t$-test or one-way ANOVA was performed for $p$ value analysis, where $<0.05$ was considered to be statistically significant. All of the statistical analyses were shown as ${ }^{*} p<0.05,{ }^{* *} p<0.01$, and ${ }^{* * *} p<0.001$ compared to the control group, respectively.

\section{Conclusions}

Our study identified that TOP I acts as an indicator in ESCC, TOP I enzyme activity suppression by HCPT attenuated cell proliferation, inhibited G2/M phase cell cycle transition, and induced apoptosis in ESCC cells in vitro and decreased ESCC PDX tumor growth in vivo. These findings mean that TOP I appears to be a valid therapeutic target and HCPT may be considered for further clinical application in ESCC.

Supplementary Materials: The following are available online at http://www.mdpi.com/2072-6694/11/12/1964/s1, Figure S1: A. The chemical structure of HCPT; B. Cytotoxicity of HCPT on normal esophageal epithelial cell SHEE. C. TOP I gene showed significant enrichment and amplified mRNA level in esophageal patient tissues compared to normal tissues in the TCGA data base, Figure S2: Annexin V and propidium iodide double staining method was used to detect apoptotic cells by flow cytometry, Figure S3: HCPT administration showed no toxicity in the PDX animal experiment, Figure S4: IHC staining in LEG110 PDX tissue specimens (100× magnification), Figure S5: The original western blot data of the expression of TOP I in different cell lines and cell cycle markers, Figure S6: The original western blot data of the expression of apoptosis markers in the four candidate cell lines, Figure S7: The original western blot data of the expression of TOP I and rH2AX in the four candidate cell lines 48 or $72 \mathrm{~h}$ after HCPT treatment.

Author Contributions: M.S, M.-H.L., and Z.D. were involved in the study concept and design, acquisition of the data, analysis and interpretation of the data, and drafting of the manuscript. M.S. and S.Y. performed the experiments. R.Z., K.L., and J.-H.S. supported the data analysis and materials. M.S., M.-H.L., J.K.K., and Z.D. wrote the manuscript. Z.D. and M.L. supervised all of the study. All authors read and approved the final manuscript.

Funding: This work was supported by grant funding from the National Natural Science Foundation of China NSFC81672767, NSFC81972839, and the Key Program of Henan Province, China Grant NO.161100510300 and Henan Provincial Government, China.

Acknowledgments: The authors thank Ran Yang, Fangfang Liu, Sen Yang, and Xiang Li in the China-US (Henan) Hormel Cancer Institute for supporting the experiments and providing materials.

Conflicts of Interest: The authors declare no conflict of interest for the current project.

\section{References}

1. Zhang, Y. Epidemiology of esophageal cancer. World J. Gastroenterol. 2013, 19, 5598-5606. [CrossRef]

2. Bollschweiler, E.; Plum, P.; Monig, S.P.; Holscher, A.H. Current and future treatment options for esophageal cancer in the elderly. Expert Opin. Pharmacother. 2017, 18, 1001-1010. [CrossRef]

3. Cesas, A.; Bagajevas, A. [Combined treatment of esophageal cancer: A review]. Medicina 2004, 40 (Suppl. 1), 161-165. [PubMed] 
4. Li, M.; Liu, Y. Topoisomerase I in Human Disease Pathogenesis and Treatments. Genom. Proteom. Bioinform. 2016, 14, 166-171. [CrossRef] [PubMed]

5. Gilmour, D.S.; Pflugfelder, G.; Wang, J.C.; Lis, J.T. Topoisomerase I interacts with transcribed regions in Drosophila cells. Cell 1986, 44, 401-407. [CrossRef]

6. Wang, J.C. Cellular roles of DNA topoisomerases: A molecular perspective. Nat. Rev. Mol. Cell. Biol. 2002, 3, 430-440. [CrossRef] [PubMed]

7. Aguilera, A.; Garcia-Muse, T. R loops: from transcription byproducts to threats to genome stability. Mol. Cell 2012, 46, 115-124. [CrossRef] [PubMed]

8. Proszek, J.; Roy, A.; Jakobsen, A.K.; Frohlich, R.; Knudsen, B.R.; Stougaard, M. Topoisomerase I as a biomarker: Detection of activity at the single molecule level. Sensors 2014, 14, 1195-1207. [CrossRef] [PubMed]

9. Holden, J.A.; Rahn, M.P.; Jolles, C.J.; Vorobyev, S.V.; Bronstein, I.B. Immunohistochemical detection of DNA topoisomerase I in formalin fixed, paraffin wax embedded normal tissues and in ovarian carcinomas. Mol. Pathol. 1997, 50, 247-253. [CrossRef]

10. Hafian, H.; Venteo, L.; Sukhanova, A.; Nabiev, I.; Lefevre, B.; Pluot, M. Immunohistochemical study of DNA topoisomerase I, DNA topoisomerase II alpha, p53, and Ki-67 in oral preneoplastic lesions and oral squamous cell carcinomas. Hum. Pathol. 2004, 35, 745-751. [CrossRef]

11. Coleman, L.W.; Perkins, S.L.; Bronstein, I.B.; Holden, J.A. Expression of DNA toposiomerase I and DNA topoisomerase II-alpha in testicular seminomas. Hum. Pathol. 2000, 31, 728-733. [CrossRef] [PubMed]

12. Gupta, D.; Bronstein, I.B.; Holden, J.A. Expression of DNA topoisomerase I in neoplasms of the kidney: correlation with histological grade, proliferation, and patient survival. Hum. Pathol. 2000, 31, 214-219. [CrossRef]

13. Coleman, L.W.; Bronstein, I.B.; Holden, J.A. Immunohistochemical staining for DNA topoisomerase I, DNA topoisomerase II-alpha and p53 in gastric carcinomas. Anticancer Res. 2001, 21, 1167-1172. [PubMed]

14. Ikeguchi, M.; Arai, Y.; Maeta, Y.; Ashida, K.; Katano, K.; Wakatsuki, T. Topoisomerase I expression in tumors as a biological marker for CPT-11 chemosensitivity in patients with colorectal cancer. Surg. Today 2011, 41, 1196-1199. [CrossRef] [PubMed]

15. Gallo, R.C.; Whang-Peng, J.; Adamson, R.H. Studies on the antitumor activity, mechanism of action, and cell cycle effects of camptothecin. J. Natl. Cancer Inst. 1971, 46, 789-795. [PubMed]

16. Kessel, D.; Bosmann, H.B.; Lohr, K. Camptothecin effects on DNA synthesis in murine leukemia cells. Biochim. Biophys. Acta 1972, 269, 210-216. [CrossRef]

17. Yuan, Z.F.; Tang, Y.M.; Xu, X.J.; Li, S.S.; Zhang, J.Y. 10-Hydroxycamptothecin induces apoptosis in human neuroblastoma SMS-KCNR cells through p53, cytochrome c and caspase 3 pathways. Neoplasma 2016, 63, 72-79. [CrossRef]

18. Fei, B.; Chi, A.L.; Weng, Y. Hydroxycamptothecin induces apoptosis and inhibits tumor growth in colon cancer by the downregulation of survivin and XIAP expression. World J. Surg. Oncol. 2013, 11, 120. [CrossRef]

19. Yang, L.; Hong, J.; Di, J.; Guo, Y.; Han, M.; Liu, M.; Wang, X. 10-Hydroxycamptothecin (HCPT) nanosuspensions stabilized by mPEG1000-HCPT conjugate: high stabilizing efficiency and improved antitumor efficacy. Int. J. Nanomed. 2017, 12, 3681-3695. [CrossRef]

20. Wang, H.; Feng, J.; Liu, G.; Chen, B.; Jiang, Y.; Xie, Q. In vitro and in vivo anti-tumor efficacy of 10-hydroxycamptothecin polymorphic nanoparticle dispersions: Shape- and polymorph-dependent cytotoxicity and delivery of 10-hydroxycamptothecin to cancer cells. Nanomed. Nanotechnol. Biol. Med. 2016, 12, 881-891. [CrossRef]

21. Hidalgo, M.; Amant, F.; Biankin, A.V.; Budinska, E.; Byrne, A.T.; Caldas, C.; Clarke, R.B.; de Jong, S.; Jonkers, J.; Maelandsmo, G.M.; et al. Patient-derived xenograft models: an emerging platform for translational cancer research. Cancer Discov. 2014, 4, 998-1013. [CrossRef] [PubMed]

22. Nakajima, Y.; Miyake, S.; Nagai, K.; Kawano, T.; Iwai, T. CPT-11 may provide therapeutic efficacy for esophageal squamous cell cancer and the effects correlate with the level of DNA topoisomerase I protein. Jpn. J. Cancer Res. 2001, 92, 1335-1341. [CrossRef] [PubMed]

23. Garcia-Carbonero, R.; Supko, J.G. Current perspectives on the clinical experience, pharmacology, and continued development of the camptothecins. Clin. Cancer Res. 2002, 8, 641-661. [PubMed]

24. Ling, Y.H.; Tseng, M.T.; Nelson, J.A. Differentiation induction of human promyelocytic leukemia cells by 10-hydroxycamptothecin, a DNA topoisomerase I inhibitor. Differentiation 1991, 46, 135-141. [CrossRef] [PubMed] 
25. Ling, Y.H.; Andersson, B.S.; Nelson, J.A. DNA topoisomerase I as a site of action for 10-hydroxycamptothecin in human promyelocytic leukemia cells. Cancer Biochem. Biophys. 1990, 11, 23-30.

26. Zou, J.; Li, S.; Chen, Z.; Lu, Z.; Gao, J.; Zou, J.; Lin, X.; Li, Y.; Zhang, C.; Shen, L. A novel oral camptothecin analog, gimatecan, exhibits superior antitumor efficacy than irinotecan toward esophageal squamous cell carcinoma in vitro and in vivo. Cell Death Dis. 2018, 9, 661. [CrossRef]

27. Huang, J.; Xu, B.; Liu, Y.; Huang, J.; Lu, P.; Ba, Y.; Wu, L.; Bai, Y.; Zhang, S.; Feng, J.; et al. Irinotecan plus S-1 versus S-1 in patients with previously treated recurrent or metastatic esophageal cancer (ESWN 01): A prospective randomized, multicenter, open-labeled phase 3 trial. Cancer Commun. 2019, 39, 16. [CrossRef]

28. Wei, Y.; Li, C.; Zhang, Y.; He, H.; Zhang, G.; Hao, X.; Liu, H.; Wang, H.; Tian, W. Hydroxycamptothecin mediates antiproliferative effects through apoptosis and autophagy in A549 cells. Oncol. Lett. 2018, 15, 6322-6328. [CrossRef]

29. Ping, Y.H.; Lee, H.C.; Lee, J.Y.; Wu, P.H.; Ho, L.K.; Chi, C.W.; Lu, M.F.; Wang, J.J. Anticancer effects of low-dose 10-hydroxycamptothecin in human colon cancer. Oncol. Rep. 2006, 15, 1273-1279. [CrossRef]

30. Min, X.; Heng, H.; Yu, H.L.; Dan, M.; Jie, C.; Zeng, Y.; Ning, H.; Liu, Z.G.; Wang, Z.Y.; Lin, W. Anticancer effects of 10-hydroxycamptothecin induce apoptosis of human osteosarcoma through activating caspase-3, p53 and cytochrome c pathways. Onco. Lett. 2018, 15, 2459-2464. [CrossRef]

31. Zhang, X.W.; Qing, C.; Xu, B. Apoptosis induction and cell cycle perturbation in human hepatoma hep G2 cells by 10-hydroxycamptothecin. Anticancer. Drugs 1999, 10, 569-576. [CrossRef] [PubMed]

32. Alagoz, M.; Gilbert, D.C.; El-Khamisy, S.; Chalmers, A.J. DNA repair and resistance to topoisomerase I inhibitors: Mechanisms, biomarkers and therapeutic targets. Curr. Med. Chem. 2012, 19, 3874-3885. [CrossRef] [PubMed]

33. Weyemi, U.; Redon, C.E.; Choudhuri, R.; Aziz, T.; Maeda, D.; Boufraqech, M.; Parekh, P.R.; Sethi, T.K.; Kasoji, M.; Abrams, N.; et al. The histone variant H2A.X is a regulator of the epithelial-mesenchymal transition. Nat. Commun. 2016, 7, 10711. [CrossRef] [PubMed]

34. Baltanas, F.C.; Weruaga, E.; Valero, J.; Recio, J.S.; Alonso, J.R. Albumin attenuates DNA damage in primary-cultured neurons. Neurosci. Lett. 2009, 450, 23-26. [CrossRef]

35. Liu, Y.; Long, Y.H.; Wang, S.Q.; Li, Y.F.; Zhang, J.H. Phosphorylation of H2A.X(T)(yr39) positively regulates DNA damage response and is linked to cancer progression. FEBS J. 2016, 283, 4462-4473. [CrossRef] [PubMed]

36. Capranico, G.; Marinello, J.; Chillemi, G. Type I DNA Topoisomerases. J. Med. Chem. 2017, 60, $2169-2192$. [CrossRef]

37. Stewart, E.; Federico, S.M.; Chen, X.; Shelat, A.A.; Bradley, C.; Gordon, B.; Karlstrom, A.; Twarog, N.R.; Clay, M.R.; Bahrami, A.; et al. Orthotopic patient-derived xenografts of paediatric solid tumours. Nature 2017, 549, 96-100. [CrossRef]

38. Shen, Z.Y.; Xu, L.Y.; Li, E.M.; Shen, J.; Zheng, R.M.; Cai, W.J.; Zeng, Y. Immortal phenotype of the esophageal epithelial cells in the process of immortalization. Int. J. Mol. Med. 2002, 10, 641-646. 